\title{
Hip Anatomy and Biomechanics Relevant to Hip Replacement
}

\author{
Romain Galmiche, Henri Migaud, \\ and Paul-E. Beaulé
}

\section{Key Points}

- Surgeons have to cope with many variations in anatomy depending on gender, geographic area, or specific diseases.

- Surgeons have to either adapt their surgical technique and/or implant positioning to deal with all anatomies or use custom-made implants.

- Implant positioning needs to be accurate as error(s) in the position of component(s) can modify essential biomechanical parameters and thus jeopardize the clinical result.

- A better understanding of the dynamic/ functional orientation of articular anatomical structures and the femoroacetabular prosthetic interplay will benefit hip arthroplasty and feed future innovations.

R. Galmiche $\cdot$ H. Migaud

Service d'orthopédie C, Hopital Salengro, Centre Hospitalier Universitaire de Lille, Lille, France e-mail: hemigaud@nordnet.fr

P.-E. Beaulé $(\bowtie)$

Orthopaedic Department, The Ottawa Hospital, Ottawa, ON, Canada

e-mail: pbeaule@ottawahospital.on.ca
Modern total hip replacement and hip resurfacing have been shown to generate good long-term clinical outcomes. Advances in materials, engineering, and improved knowledge in joint anatomy and biomechanics, have enabled this success. Successful hip prosthetic surgery relies on a proper understanding of the hip anatomy and its biomechanics. In this chapter, we will review these essential points.

\subsection{Normal Hip Biomechanics}

Understanding of the human gait has progressed since the early methods of chronophotography by Etienne-Jules Marey, which enabled capture of human movement. Expansion on this through advancement in technology, such as infrared cameras, electromyographs, and force platforms, has led to a greater understanding not only of human locomotion, but also the effects our surgery has. The importance of hip biomechanics has become more and more prominent with the development of gait laboratories giving us a more accurate, but also more complex, view of the hip's in vivo function.

\subsubsection{Kinematics}

Hip motion is allowed in three planes (sagittal, frontal, and transverse) due to its ball-and-socket 
configuration. Nevertheless, some authors have described the femoral head with a conchoid (or ellipsoid) shape [1]. This particular shape makes the joint less likely to sublux when compared to a true ball-and-socket joint. Moreover, this shape may contribute to generation of the optimal stress magnitude and distribution [2]. In the same manner, the horseshoe geometry of the acetabular cartilage has been shown to optimize the contact stress distribution. Thus, through the acknowledgment of these anatomic features, we immediately understand that allowing mobility while maintaining stability is the first challenge a prosthetic spherical implant faces.

The sagittal plane portrays the greatest passive range of motion: flexion, on average, can reach $100^{\circ}$ (extended knee) and $140^{\circ}$ (flexed knee, due to the hamstring release). Extension is $15^{\circ}-20^{\circ}$. In the frontal plane, the range of abduction is from $10^{\circ}$ to $45^{\circ}$, whereas the range of adduction is $10^{\circ}-30^{\circ}$. The external rotation reaches $60^{\circ}$ and the internal rotation $30^{\circ}$, but it can go further when the hip joint is flexed due to the release of the soft tissues (up to $90^{\circ}$ for external and $60^{\circ}$ for internal rotation). However, these figures are subject to interindividual variation. Gender, age, individual patient anatomy (femoral neck angle, femoral neck offset, acetabulum version ...), and level of physical activity are features that can alter the hip range of motion. For example, a subject with a coxa valga tends to exhibit a better abduction peak angle than a coxa vara subject, due to the delayed impingement between the femoral neck and the acetabular labrum.

As an orthopedic surgeon, it is important to know the values of hip motion involved in activities of daily living. For example, tying shoe laces with feet on the floor will require up to $125^{\circ}$ hip flexion, $19^{\circ}$ external rotation, and $15^{\circ}$ of abduction; ascending stairs will require a mean hip flexion of $70^{\circ}$, whereas descending them needs $35^{\circ}$. Gait is characteristic of the human species. This is a succession of imbalance phases that is actually much more complex than the human eye can see. Measurements in the sagittal plane (Fig. 2.1) show that the hip joint is maximally flexed $\left(35^{\circ}-45^{\circ}\right)$ during the late swing phase of gait, as the limb moves forward for heel strike. Then, the hip extends as the body moves forward, and the extension peak is reached at heel-off. The frontal and transverse planes are also involved. Abduction occurs during the swing phase of gait and reaches a maximum just after toe-off. At heel strike, the hip joint reverses into adduction and keeps it during the entire stance phase. The hip joint is externally rotated during the swing phase and, to provide a fitted angle for the foot strike, the hip rotates internally. This internal rotation is gradually lost as the contralateral hip moves forward. One should also consider the motion of the pelvis (in sagittal, axial, and frontal planes) during the walking sequence. Pelvic motion is highly variable between individuals and its amplitude depends on multiple parameters, such as walking speed, pelvic and hip anatomy (e.g., width of pelvis), flexibility of the spine and the hips, etc. This pelvic motion probably has a significant influence on the hip biomechanics and the risk of degeneration. One must acknowledge that the pelvis undergoes axial rotation (about $8^{\circ}$ ) as the leg moves forward. There is a heightening of the hemi-pelvis before toe-off as well (corresponding to a $5^{\circ}$ rotation in the frontal plane), introducing the concept of "pelvic vertebra" asserted by Jean Dubousset. These motions require further investigation, given they are highly variable between individuals and may produce deleterious effects on bearing components (edge loading, impingement) in dynamic situations [3].

\subsubsection{Kinetics}

Joint reaction forces are the forces generated within the joint in reaction to forces acting on the joint. For the hip, it is the result of the need to balance the moment arms of the body weight and abductor tension in order to keep a leveled pelvis. The hip contact forces are then a combination of ground reaction force to body weight, and of internal muscle contraction forces. The resultant hip reaction forces can be calculated either in vivo, by strained-gauged prosthesis, or by analytical approaches (2D models or more sophisticated 3D 
Fig. 2.1 Hip's movements in the three dimensions during a normal gait cycle. Red vertical line represents heel-strike and green vertical line is toe-off. $A$ Stance phase, $B$ Swing phase

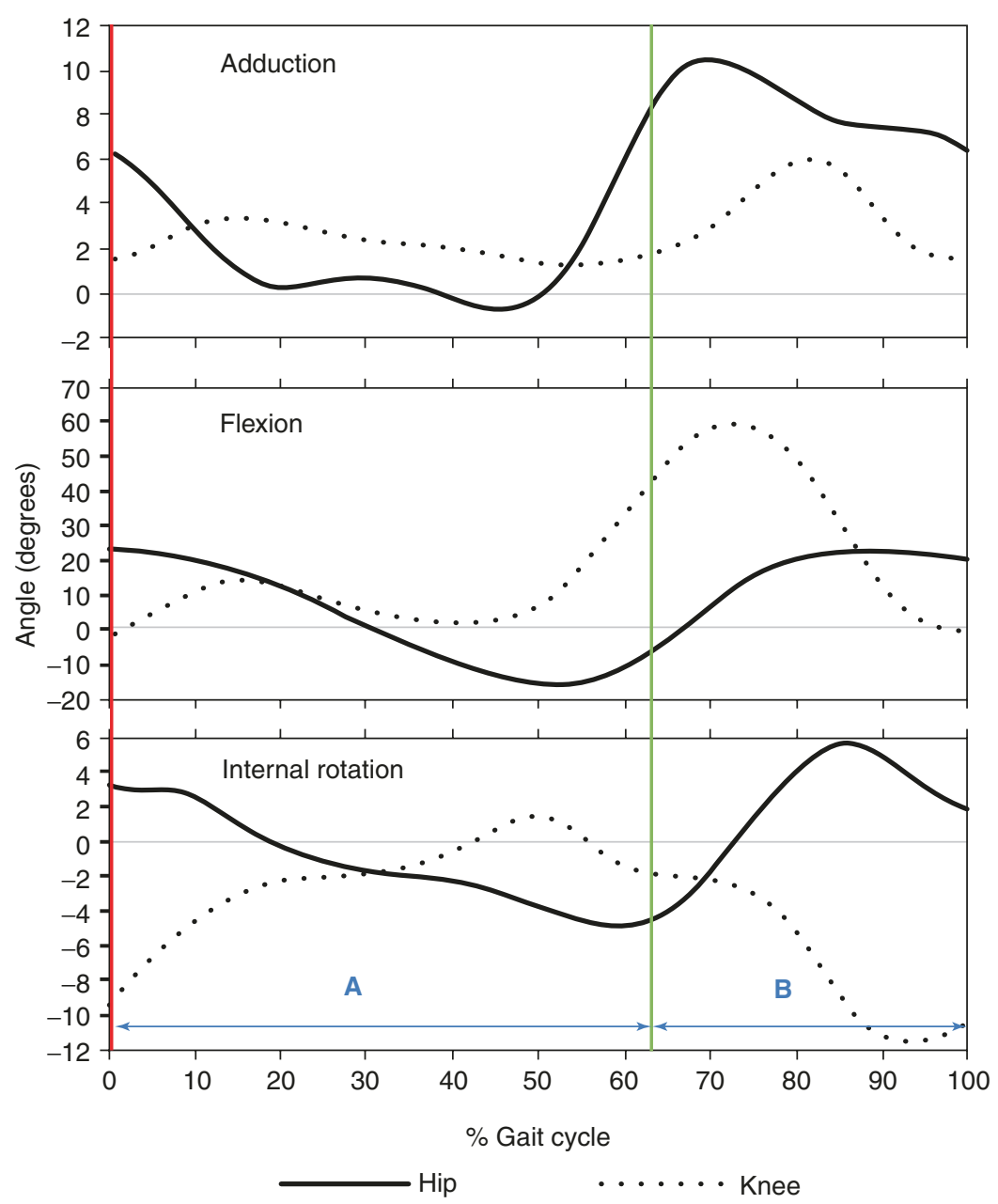

(internal moment), in order to keep a poised pelvis. Since the effective lever arm of abductor muscles is considerably shorter than the effective lever arm of body weight, the combined force of abductors must be a multiple of body weight. It ensues peak hip joint forces can reach 1.8 to 4.3 times body weight during gait [4]. These numbers could rise to eight times body weight for activities like running or skiing. This highlights how these forces will play a first rank role in the selection of components, implantation, wear, and durability. On the femoral head, maximum contact pressures occur at the supero-anterior area during walking, whereas for the acetabulum, the supero-posterior zone is more exposed to constrain. When moving from standing to sitting position or from sitting to standing position, the contact pressure is higher 


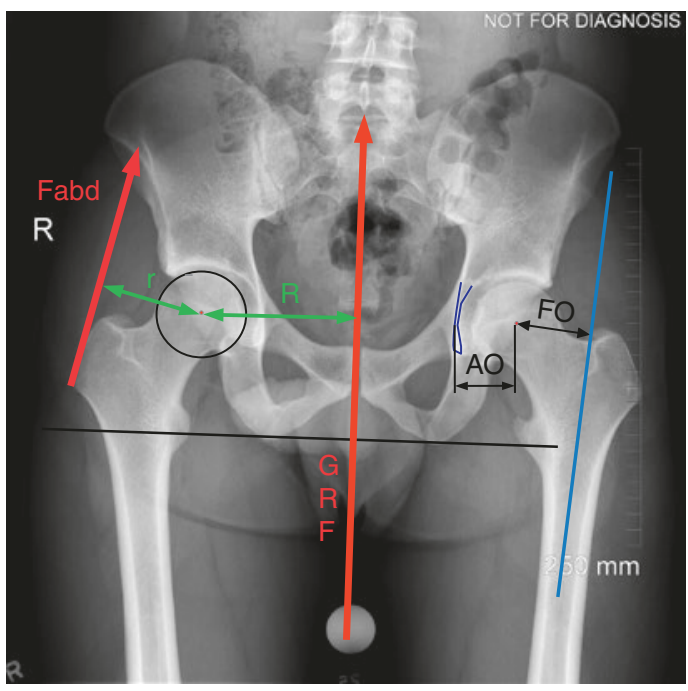

Fig. 2.2 AP pelvis X-rays. Fabd Abductor muscle force, GRF Ground Reaction Force, $F O$ Femoral Offset, $A O$ Acetabular Offset, $r$ Abductor lever arm, $R$ Body weight lever arm

mainly due to the smaller contact area at the edge of the posterior horn of the acetabulum. Indeed, as the hip is flexed, the contact area moves posteriorly. There are typically two hip resultant force peaks during the stance phase: a first one in early stance and a second one in late stance. One should also consider forces in the horizontal plane, which have been barely investigated. These forces may jeopardize efforts to optimize bearing components' behavior, as their influence is not fully understood.

Many parameters influence the intensity and repartition of the resultant hip joint contact forces. From a mechanical point of view, the abductor lever arm (tied to the neck-shaft angle and neck length) and the body-weight lever arm (tied to pelvis width) are two important parameters, particularly because they can easily be modified by THR surgery. The magnitude of body weight is also significantly influential. An increase in the abductor lever arm will lead to a decrease of the abductor's force needed to maintain a horizontal pelvis. It will tend to decrease the hip joint reaction forces. In the same way, a wider pelvis increases the body weight lever arm, and so will increase the joint contact forces during the one-legged stance. All of this is true in reverse, and applicable to prosthetic hips as well. As we suggested at the beginning of this chapter, the native hip joint is actually more complex than a simple ball-and-socket model. Cartilage and bone elasticity, a slight lack of congruency due to acetabular deformation the more the hip is loaded, the conchoid femoral head shape, as well as the acetabular and femoral neck orientation are parameters playing intricate roles in the hip contact forces' magnitude and repartition. Nonspherical shapes allow rolling movements in addition to sliding movements, which are logically the only ones found in a perfect ball-and-socket model. Thus, studies showed these conchoid or ellipsoid shapes contributed to optimal stress magnitude and repartition. In the same manner, cartilage elasticity, which is lost in an arthroplasty surgery, optimizes load transfer. In vitro, a decrease in acetabular anteversion leads to a dramatic increase in the hip's load, as reported by Sanchez Egea. A similar result is observed when decreasing femoral anteversion or neckshaft angle [5]. However, one should consider the in vivo interaction between the femoral and acetabular anteversion-inclination. Indeed, it is more relevant to look at the interplay between acetabulum and proximal femur orientation. It introduces the concept of combined version, which recommends that the sum of the stem and cup anteversion values approximates $37^{\circ}$ [6]. Accurate combined anteversion is more likely to result in a harmonious interaction between the femoral head and the cup, with no impingement throughout the entire range of body positions.

In the prosthetic joint, the femoral head diameter, articular clearance, and cup orientation are other important parameters influencing the head/acetabulum contact area (or contact patch), and therefore the hip joint contact forces. For a bigger head diameter, one would expect a larger contact patch between head and cup. However, the contact patch size is closely tied to the inner diameter of the cup, as well as defining the clearance. Thus, too high a clearance will reduce the contact patch area, potentially leading to a high wear rate. On the other hand, low clearance hips have a more conformal contact and a larger contact patch, which decreases the distance between the edge of the contact patch and the rim of the cup, thereby increasing the risk of edge loading and wear. Edge loading occurs 
when the contact patch between the head and cup extends over the cup rim, which results in a large increase in local pressure, disruption of the lubrication mechanism, and increased wear. Clearance is now known to be an important factor in edge loading phenomenon [7]. This consideration is of high importance specifically for large diameter MoM bearings. A cup abduction angle of $45^{\circ}$ or less is recommended to avoid excessive wear. It is of particular importance for MoM resurfacing in order to avoid edge-loading phenomenon. The effect of cup anteversion on wear is less straightforward and should be considered alongside the femoral version [8]. Nevertheless, modifying the cup inclination and/or anteversion will influence both the anterosuperior and the posteroinferior cup-head contact areas in opposite ways. For hard on smooth bearing couples, liner wear rates for 22,28 or $32 \mathrm{~mm}$ heads do not vary significantly. Nevertheless, volumetric wear increases with head size, as it impacts the sliding distance between bearing components.

\subsection{Variability in Hip Anatomy}

In order to restore physiological hip biomechanics, THR surgery often aims to respect the patient's individual anatomy. However, hip anatomy is subject to a high interindividual variability. Immediately we understand the surgical difficulties related to this, in particular our capacity to restore the infinite natural variation with prosthetic implants.

Are there gender differences? In addition to the age, weight, and height, which play a major role in interindividual differences, there are other elements linked to anatomical variation. Gender is the first parameter associated with anatomical variability [9-13], with the pelvis exhibiting specific characteristics depending on gender; in females the pelvis is wider and the acetabulum generally deeper with greater anteversion when compared to males: $18^{\circ}$ vs. $21^{\circ}[14]$ and inclination $38.5^{\circ}$ vs. $36^{\circ}$ [15] (Fig. 2.3). These differences are partially explained by the developmental

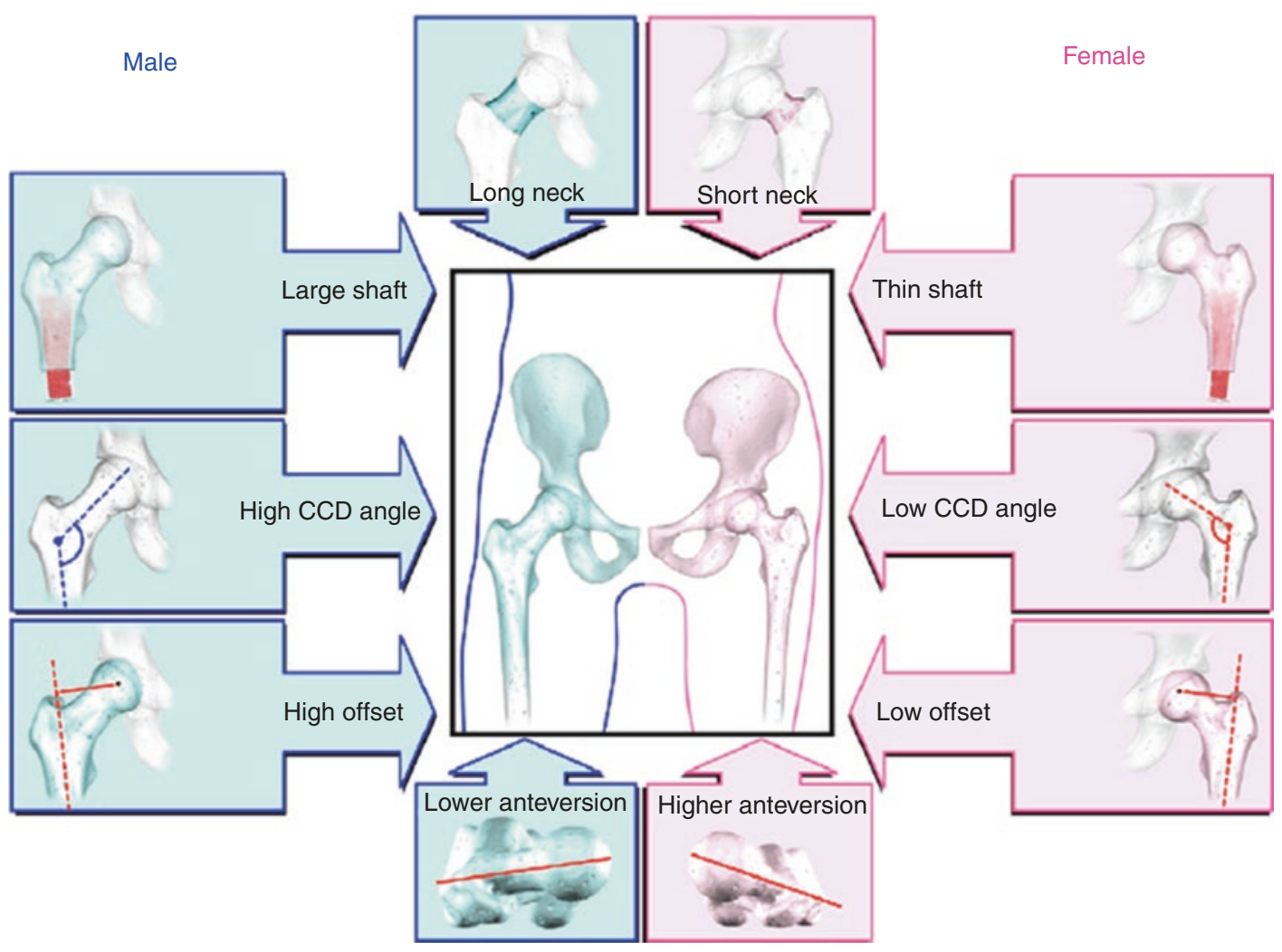

Fig. 2.3 Main gender differences regarding hip morphology [14] 
response to the need to give birth, where the birth canal is wider. However, with the broader pelvis, the body-weight lever arm is increased, which is associated with a deeper acetabulum (up to coxa profunda), thus reducing the body-weight lever arm. On the femoral side, females have a smaller femoral head diameter (adjusted for height and weight), a greater femoral anteversion, thinner femoral shaft, and a lower femoral shaft-neck angle with an associated smaller femoral offset when compared to men: $48 \mathrm{~mm}$ vs. $55 \mathrm{~mm}$. Another key difference is the lower bone mineral density seen in females, especially after menopause, increasing the risk of peri-prosthetic fracture. These anatomical differences and their impact on joint replacement were quite evident with metal-on-metal hip resurfacing, where the smaller head size and acetabular orientation lead to a higher risk of failure. Differences are also found in the range of motion: women exhibit a greater peak hip flexion and internal rotation (hip at $90^{\circ}$ flexion), whereas men show greater peak hip extension and external rotation.

Are there racial/ethnical variants? A number of studies, mainly from the 1950s, reported wider pelvises (both pelvic inlet and outlet) in the Caucasian population compared to the African population. This was thought to be associated with a higher geographic latitude. Many factors can explain these differences in pelvic shape around the world. The climate adaptation theory, which claims that narrower pelvises are seen in lower latitudes, while wider pelvises are seen in more northern areas in order to save heat and energy, has recently gained attention [16]. It questions the original, and until now, widely accepted theory that pelvic shape is the result of the evolutionary compromise (obstetric dilemma) between efficient bipedal locomotion and the safe parturition of a neonate. In reality, it is probable that multiple factors influence pelvic shape, and that environment and lifestyle (e.g., alimentation, activities) are likely to be equally as responsible as ethnical/geographic factors. For example, with regard to the neck-shaft angle, the activity level is a strong determinant, given that the neck tends to become more varus as the activity level increases [9, 10]. Finally, racial differences in sacral geometry and spino-pelvic alignment have already been reported [17] in addition to gender differences [18].

Is there a "normal" hip? Independent of gender or ethnic origin, some constitutional variations are found among us, and they sometimes lead to a pathological process. Acetabular retroversion on plain X-ray can affect $6 \%$ of hips in a healthy population and up to $20 \%$ and $42 \%$ in osteoarthritis and Legg-Perthes-Calve cohorts, respectively $[19,20]$. Coxa profunda may affect 5-20\% of the whole population [21]. Acetabular retroversion represents a particular form of hip dysplasia, characterized by abnormal posterolateral orientation of the acetabulum. This pathophysiology predisposes the individual to subsequent anterior impingement of the femoral neck upon the anterior acetabular margin and fibrous labrum. Similarly, developmental hip dysplasia has a prevalence of $3.6-4.3 \%$ in the healthy adult population [22]. These pathologies can present a technical challenge for prosthetic surgery, particularly in their extreme states, which may involve a combination of hip dislocation, leg length discrepancy, posterior-superior acetabulum defect, and acetabulum retroversion. Dynamic study of the pelvis-femur relationship can complicate the notion of anteversion and retroversion. Firstly, pelvic tilt values in the standing position differ from one individual to another, with an average of $12^{\circ}$ in the Caucasian population with standard deviation around $6^{\circ}$ [17]. In addition, the pelvic tilt varies between supine, standing, and sitting positions, thus modifying the functional orientation of the acetabulum; this is enabled by the lumbar spine sagittal flexibility [23-25]. Thus, one has to know that these variations cannot be ignored, especially for cup positioning: in the supine position, the pelvis tilts anteriorly, which decreases anteversion of the acetabular component, while in the standing and sitting positions, the reverse happens and anteversion is increased $[24,26]$. With regard to the femoral neck-shaft angle, Boese et al. reported in 
a review article that the interindividual differences in the healthy population can range from $98^{\circ}$ to $160^{\circ}$ and from $115^{\circ}$ to $155^{\circ}$ in the osteoarthritic population [27]. This in turn affects the femoral offset, which is directly linked to the neck-shaft angle and femoral neck length. Similarly, femoral torsion can also vary, resulting in anteversion or retroversion of $>40^{\circ}$; this may justify the need for $3 \mathrm{D}$ templating given regular AP pelvis radiographs are less accurate in assessing femoral torsion and medial offset. In addition to these key reconstruction parameters, the endofemoral canal can take the form of several different shapes, as measured by the femoral flare index and the cortico-medullary index (Fig. 2.4) [28]. This is of particular relevance in cementless fixation, where the need for a close bone-prosthesis interface is essential. One may favor hip resurfacing in some of these more complex situations (Fig. 2.5). In addition, anatomical variations exist in the vascularization of the femoral head, particularly the role of the inferior gluteal and medial femoral circumflex arteries, which are important to consider in hip resurfacing arthroplasty [29]. Each hip is therefore defined by an infinite combination of anatomical and geometric parameters, in addition to its functional capacity.
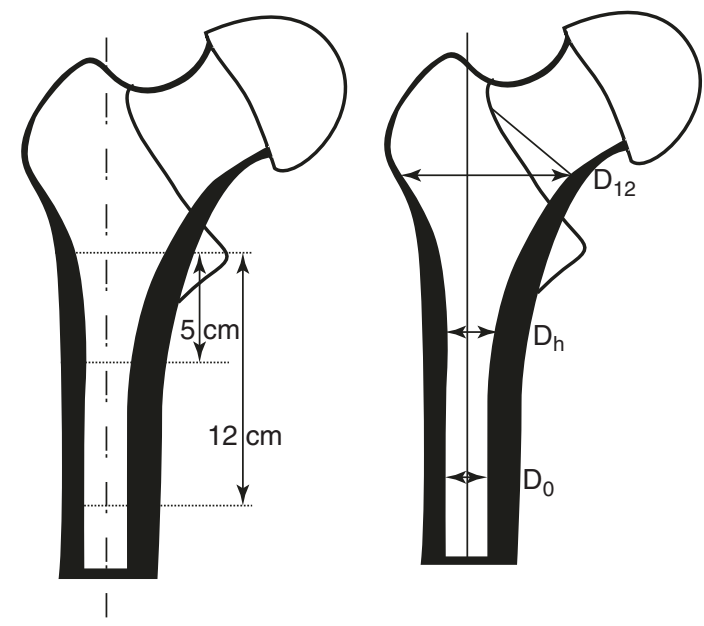

Fig. 2.4 Femoral Flare Index = D0/D12; Corticomedullary Index $=$ Medial + Lateral cortical thickness/D0
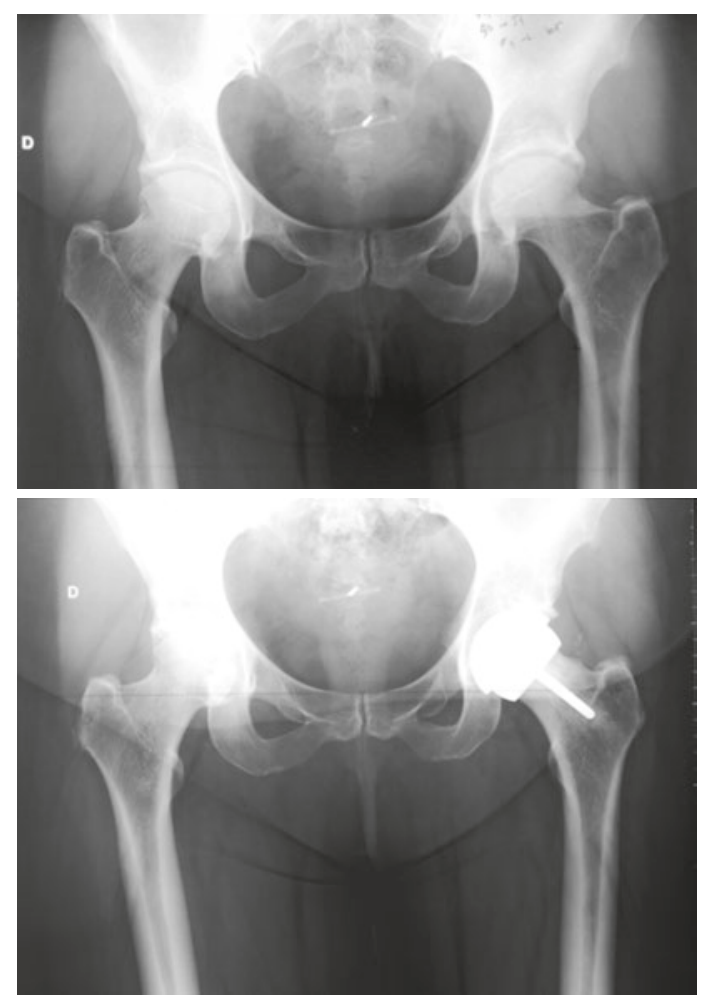

Fig. 2.5 Example of a 50-year-old woman who portrays an extra-small Femoral Flare Index. As, restoration of the femoral offset would have been difficult with a regular femoral stem, resurfacing provides a predictable anatomical reconstruction

\subsection{Anatomy Modifications Affecting Clinical Results}

\subsubsection{Relative to Components' Positioning}

Hip replacement and resurfacing aim to achieve sustainable restoration of hip mobility without pain. Component positioning plays a role in every aspect of the clinical outcomes: function, wear rate, occurrence of complication, and components' life span.

Center of rotation. In the frontal plan, the acetabular offset defines the mediolateral location of the center of rotation (Fig. 2.2). By medializing the acetabular component, one reduces 
the body moment arm, thus decreasing the amount of force generated by the abductors resulting in a decrease in the joint reaction force. However, if done excessively, this can reduce abductor muscle tension, which may need to be adjusted with the femoral offset. Thus, in order to restore the global offset (or the sum of the acetabular and femoral offset) in order to maintain adequate abductor muscle tension, you have to increase the femoral offset [30]. If the global offset decreases, the abductors' tension drops and becomes unstable. Conversely, if the combined offset increases, the abductors' tension will be excessive, potentially yielding more trochanteric pain, and there will be more torque force on the femoral stem, potentially leading to loosening [31] and peri-prosthetic fracture issues. When the center of rotation is lateralized compared to the native one, the femoral offset has to be reduced in order to conserve the original relationship between the greater trochanter and the pelvis. However, reduction of the femoral offset directly reduces the abductor lever arm, meaning abductor muscles have to produce more force in order to stabilize the pelvis. This consequently increases the joint reaction forces and wear at bearing surfaces. A geometric technique has been described to find the theoretical center of rotation by using the $\mathrm{U}$ landmark; a constant ratio has been observed between lateral position and height of the center of rotation and pelvis' width and height. Thus, it can be useful in cases where both sides are pathological [32].

Cup orientation. Hip prosthetic surgery entails removing the labrum and decreasing the head size for a THA case. Given this, the hip stability cannot remain the same. The native acetabulum covers the femoral head at $170^{\circ}$, whereas a prosthetic acetabular cup portrays a $180^{\circ}$ design $\left(170^{\circ}\right.$ or less for resurfacing cups). Thus, cup positioning has to consider stability and avoid prosthetic impingement. Cup positioning must also take into account the influence on wear. Two parameters have to be considered for the position of the cup around its own center of rotation: its inclination and its anteversion. The cup inclination will play a role in the edge-loading phenomenon by influencing the CPCR (contact patch center to rim distance) and CPER (contact patch edge to rim distance). The less inclined the cup is, the greater CPCR is in order to avoid edge loading. In addition, edge loading affects the lubrication regime and behavior of synovial fluid, which will further increase wear rates. With regard to cup anteversion, it is a major feature for prosthesis' stability, as a more anteverted cup will tend to avoid posterior dislocation. Having said that, cup version is not the only determinant of hip stability, as other factors (e.g., surgical approach, prosthetic design, head diameter, prosthetic neck anteversion) play a major role. Several methods have been described to position the cup during surgery and used either intra- (posterior and anterior rim, transverse acetabular ligament) and/or extra(anterior pelvic plane) articular anatomical landmarks. Lewinnek initially described the safe cup implantation zone in order to reduce the risk of dislocation. It was defined as a $15^{\circ} \pm 10^{\circ}$ anteversion and a $40^{\circ} \pm 10^{\circ}$ lateral opening. However, better understanding of the lumbo-pelvic sagittal kinematics and the functional acetabular orientation has challenged the value of the Lewinnek safe zone when compared to a more personalized individual "safe zone" [33, 34]. Arthroplasty is progressively switching from a systematic to a patient-specific approach $[33,34]$.

Femoral stem positioning. Error in the implantation of the femoral stem will alter the restoration of the native hip anatomy and biomechanics. It's positioning in varus or valgus may either increase or decrease the femoral offset and abductor lever arm and potentially hinder optimal clinical outcomes. In the same manner, error in adjusting the stem version will modify the lever arms, potentially induce impingement, and affect the location of the contact patch between the head and acetabulum. Above all, the leg length discrepancy is clearly directly linked to the femoral stem cranio-caudal positioning and represents the second highest cause of litigation among US surgeons [35].

\subsubsection{Relative to Components' Features}

As aforementioned, component positioning can modify the native anatomy and biomechanics, but in addition, the components themselves differ 
from the native anatomy features. On the femoral side, switching from a conchoid shape to a completely spherical shape changes anatomy. It has been shown that this special conchoid shape makes the joint less likely to sublux when compared to a true ball-and-socket joint. Furthermore, these shapes may contribute to the optimal stress magnitude and distribution. Adding to that the fact that the labrum is removed in prosthetic hip surgery, this emphasizes how anatomical concepts can be modified during hip surgery. In normal hip joint biomechanics, the labrum is crucial in retaining a layer of pressurized intra-articular fluid for joint lubrication and load support/distribution. Its seal around the femoral head is further regarded as a contributing factor to hip stability through its suction effect [36]. It is important in increasing the contact area, thereby reducing contact stress as well. For the head diameter, usual prosthetic head size ranges from 22 to $36 \mathrm{~mm}$, whereas the native average head size is $49 \mathrm{~mm}$ for women and $53 \mathrm{~mm}$ for men. The main drawback of this size reduction is the stability impairment. It is well known nowadays the dislocation rates decrease when the femoral head size increases. Reduction of the femoral head size could also have a negative impact on proprioception. Another point is the head-neck offset modification; its main impacts are on the range of motion and the prosthetic impingement risk (additionally influenced by both cup positioning and femoral stem anteversion). Prosthetic impingement could lead to cup loosening (by increasing torque on the cup), prosthetic instability, increased wear, and liner fracture. A larger femoral head will offer a better head-neck offset and thus will reduce the risk of prosthetic impingement, in addition to facilitate a better range of motion. Several authors showed that this risk becomes negligible with a prosthetic femoral head $\geq 32 \mathrm{~mm}[37,38]$. The medial femoral offset is dictated by the femoral stem design, and its restoration is intimately tied to the prosthetic portfolio available. Another element to consider is the modification of Young's modulus of elasticity inside the femoral shaft by using a $15-20 \mathrm{~cm}$ length titanium or $\mathrm{CoCr}$ alloy stem. It raises questions about proprioception modifications and above all, introduces the stress-shielding concept. Mini-stem designs and resurfacing could lead to better proprioception by enabling natural femoral shaft deformation and elasticity, primarily for patients practicing impact sports (running). Nevertheless, scientific ways to evaluate that kind of hypothesis are limited. Moreover, using a conventional femoral stem, a part of the stress force is directly transferred to the femoral shaft, bypassing the metaphysis area. Nonnatural bone remodeling phenomenon is subsequently involved, modifying the initial bone architecture. Hip resurfacing avoids these drawbacks by preserving a close to natural stress distribution.

\subsection{When Is it Safe to Recreate the Constitutional Hip Anatomy?}

Osteoarthritis can be primary or secondary. In primary cases, the patient's anatomy is deemed as normal and may be reproduced, whereas for some cases of secondary osteoarthritis, the patient's hip anatomy is considered abnormal, with articular cartilage damage being a consequence of impaired hip biomechanics. As Karimi et al. [39] mentioned, we have to be even more careful with the younger population as the percentage of secondary arthritis is higher among this group. The answer to the question "which constitutional hip anatomies may safely be restored when performing hip replacement?" still remains elusive.

It is important to be aware that most abnormal hip anatomies (CAM effect, abnormal combined anteversion causing pincer femoro-acetabular impingement, roof insufficiency) responsible for hip degeneration are automatically corrected when anatomically implanting modern component designs. Nonetheless, severely abnormal hip anatomy (e.g., atypical femoral and/or acetabular anteversion, protrusio acetabulum) may need to be corrected as they are potentially biomechanically inferior. For individuals with abnormal femur and/or acetabulum anteversion, one should: (1) assess the individual spine-hip relationship to understand the functional acetabular orientation and (2) perform 3D planning with simulated hip ROM, in order to predict the optimal implant 
positioning and design. For a protrusio acetabuli or a dysplastic acetabulum with roof deficiency, an appropriate center of rotation will have to be reconstructed, which will diverge from the constitutional one. Whatever the severity of the protrusio, the ilio-ischial line remains a good landmark for reconstructing the center of rotation; the goal is to lateralize the hip center in order to avoid instability and prosthetic neck-bone impingement. Any severe defect of the acetabular roof should be corrected by either bone grafting or metallic augmentation, plus or minus a reinforcement ring.

The proximal femoral anatomy is highly variable between individuals. Coxa vara and coxa valga, as well as unusual femoral offset, are anatomic features that generally have to be respected. Aside from cases of developmental hip disease, any modifications of the proximal femur anatomy are likely to hinder optimal clinical outcomes [40]. The surgical solutions to restore these parameters when facing extreme values are detailed below in this chapter. Nevertheless, hip resurfacing appears to be the best means of keeping the natural hip anatomy with regard to the femoral side, although it is technically strenuous. When performing hip resurfacing, special attention must be paid to the constitutional head-toneck offset of hips, which have degenerated due to cam-type impingement. In order to obtain good and steady results in these cases, surgical correction of this bone impingement is required alongside resurfacing. This is achieved by maximizing and/or moving anteriorly the femoral component, plus or minus osteoplasty of the anterosuperior part of the neck.

Hip osteoarthritis often leads to a true leg length discrepancy due to articular surface wear. One should not ignore the possibility of an additional functional leg length discrepancy from a fixed pelvis obliquity and/or hip stiffness. A sound understanding of these mechanisms is essential to avoid any errors in reestablishing the correct leg length. The length of the femur is a parameter that can be reliability restored by adjusting the craniocaudal positioning of the stem and the head neck length. Nonetheless, we have to bear in mind that our surgery can sometimes lead to functional leg length discrepancy by lengthening the hip (voluntary in case of high-grade dysplasia) or by increasing the global offset (voluntary in case of protrusio). These functional discrepancies are often resolved within a year after surgery as soft tissues progressively remodel.

\subsection{Limitations of Implants in Restoring Native Hip Anatomy}

There are two kinds of limitations for prosthetic implants. Firstly, there is the compulsory limit set by the portfolio size. The size scale is globally limited to the values represented within the $90 \%$ in the center of the bell curve. For most femoral stems, the femoral offset increases with the size of the implant; an issue can arise when the patient displays a mismatch between the femoral canal width and the femoral offset (Fig. 2.6). Nevertheless, modularity, especially modular-neck

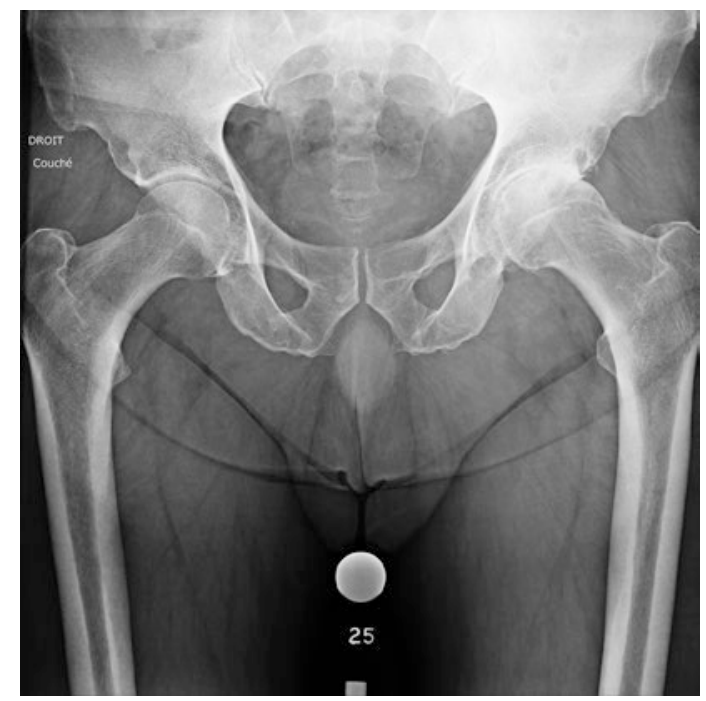

Fig. 2.6 In this case, the patient, a male of 72 years old, portrays an out-of-the-range femoral offset and neck's length whereas he depicts a very narrow femoral canal. Without templating, the error would be to try putting on a regular stem: the chosen size would be necessarily a small one because press fit would be quickly acquired in the femoral canal. In consequence, the femoral offset, tied to the size of the femoral stem would not be restored, as it should. Decision to use a custom femoral implant has been taken (Fig. 2.7) 


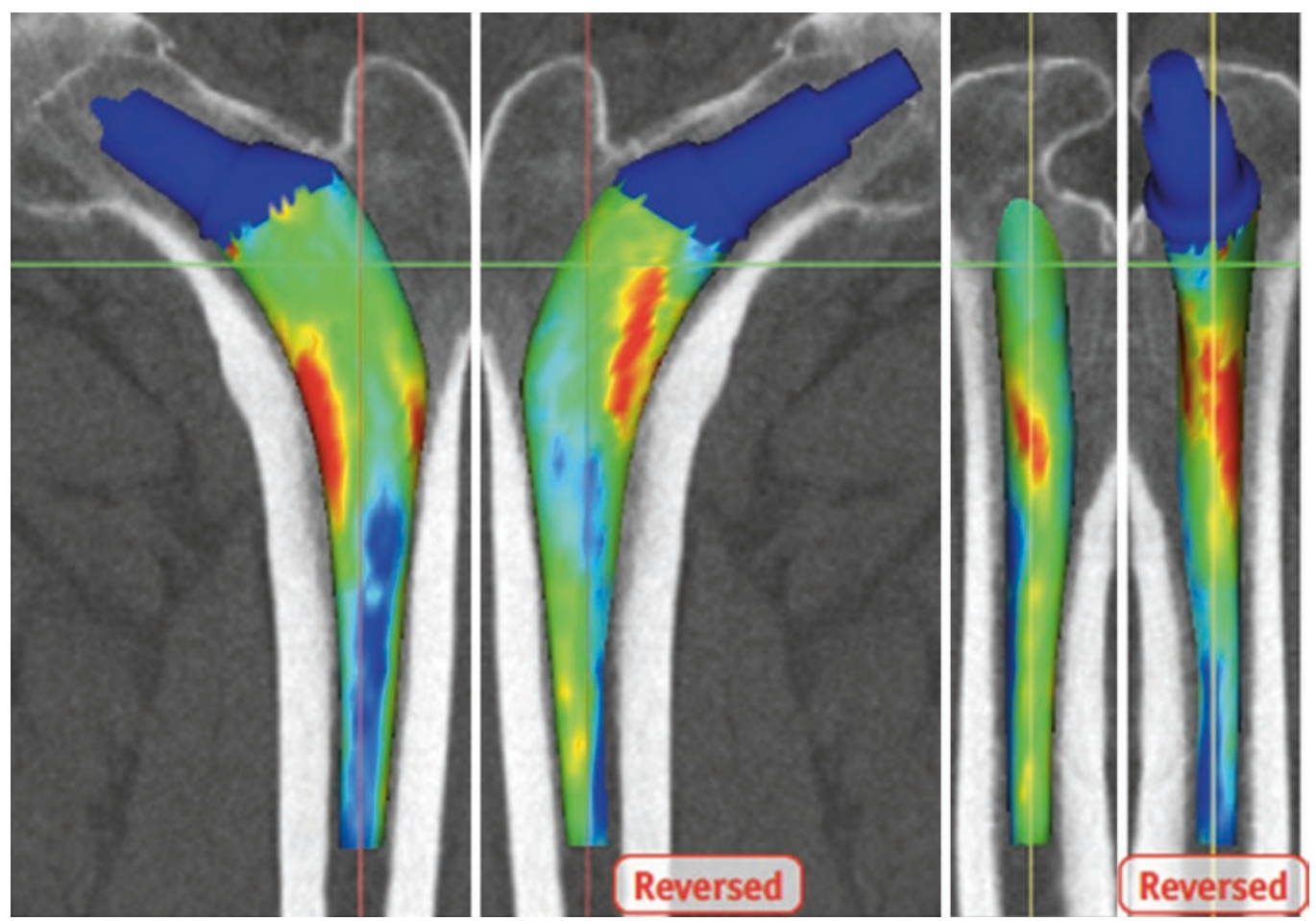

Fig. 2.7 Custom femoral implant designed for patient in Fig. 2.5

femoral stems, has provided a solution in some cases over the last few decades. Secondly, there is an engineering limitation: excessively long femoral neck, for example, cannot be safely reproduced by a manufactured implant due to the risk of prosthetic neck fracture. This means that even custom implants can encounter difficulties when dealing with extreme anatomies. As represented in Fig. 2.7, custom implants sometimes enable us to deal with abnormal anatomy, such as extreme coxa vara. However, the cementless fixation mode remains the same (even if the implant design fits the endo-femoral canal), while the torque that the femoral stem has to tolerate becomes higher. Perhaps we should monitor the long-term life span of custom implants made for this kind of use.

\subsection{Conclusion}

With the technical possibilities currently offered by materials and prosthesis engineering, the need to consider interindividual variation in hip anat- omy is gaining recognition among the orthopedic community. There are more and more technical solutions to suit all femoral and acetabular shapes. In parallel, the recent understanding of hip biomechanics, with regard to the dynamic relationship between the femur and acetabulum, has altered our classic view of the anatomy. These concepts present a challenge for each orthopedic surgeon and should be a central point in our future research.

\section{References}

1. Menschik F. The hip joint as a conchoid shape. J Biomech. 1997;30(9):971-3.

2. Gu D-Y, Hu F, Wei J-H, Dai K-R, Chen Y-Z. Contributions of non-spherical hip joint cartilage surface to hip joint contact stress. Conf Proc IEEE Eng Med Biol Soc. 2011;2011:8166-9.

3. Dujardin F, Selva O, Mejjad O, Pasero D, Piraux JL, Thomine JM. Intra and interindividual variations of pelvic mobility in normal adult walk. Rev Chir Orthop Reparatrice Appar Mot. 1995;81(7):592-600. 
4. Bergmann G, Deuretzbacher G, Heller M, Graichen F, Rohlmann A, Strauss J, et al. Hip contact forces and gait patterns from routine activities. J Biomech. 2001;34(7):859-71.

5. Sánchez Egea AJ, Valera M, Parraga Quiroga JM, Proubasta I, Noailly J, Lacroix D. Impact of hip anatomical variations on the cartilage stress: a finite element analysis towards the biomechanical exploration of the factors that may explain primary hip arthritis in morphologically normal subjects. Clin Biomech. 2014;29(4):444-50.

6. Dorr LD, Malik A, Dastane M, Wan Z. Combined anteversion technique for total hip arthroplasty. Clin Orthop. 2009;467(1):119-27.

7. Underwood RJ, Zografos A, Sayles RS, Hart A, Cann P. Edge loading in metal-on-metal hips: low clearance is a new risk factor. Proc Inst Mech Eng $\mathrm{H}$. 2012;226(3):217.

8. Hart AJ, Ilo K, Underwood R, Cann P, Henckel J, Lewis A, et al. The relationship between the angle of version and rate of wear of retrieved metal-on-metal resurfacings: a prospective, CT-based study. J Bone Joint Surg Br. 2011;93(3):315-20.

9. Anderson JY, Trinkaus E. Patterns of sexual, bilateral and interpopulational variation in human femoral neck-shaft angles. J Anat. 1998;192(Pt 2):279-85.

10. Gilligan I, Chandraphak S, Mahakkanukrauh P. Femoral neck-shaft angle in humans: variation relating to climate, clothing, lifestyle, sex, age and side. J Anat. 2013;223(2):133-51.

11. Milligan DJ, O'Brien S, Bennett D, Hill JC, Beverland DE. The effects of age and gender on the diameter of the femoral canal in patients who undergo total hip replacement. Bone Jt J. 2013;95-B(3):339-42.

12. Tannenbaum E, Kopydlowski N, Smith M, Bedi A, Sekiya JK. Gender and racial differences in focal and global acetabular version. J Arthroplast. 2014;29(2):373-6.

13. Wang SC, Brede C, Lange D, Poster CS, Lange AW, Kohoyda-Inglis C, et al. Gender differences in hip anatomy: possible implications for injury tolerance in frontal collisions. Annu Proc Assoc Adv Automot Med. 2004;48:287.

14. Nakahara I, Takao M, Sakai T, Nishii T, Yoshikawa H, Sugano N. Gender differences in 3D morphology and bony impingement of human hips. J Orthop Res Off Publ Orthop Res Soc. 2011;29(3):333-9.

15. Traina F, De Clerico M, Biondi F, Pilla F, Tassinari E, Toni A. Sex differences in hip morphology: is stem modularity effective for total hip replacement? J Bone Joint Surg Am. 2009;91(Suppl 6):121-8.

16. DeSilva JM, Rosenberg KR. Anatomy, development, and function of the human pelvis. Anat Rec Hoboken NJ. 2017;300(4):628-32.

17. Endo K, Suzuki H, Nishimura H, Tanaka H, Shishido T, Yamamoto K. Characteristics of sagittal spinopelvic alignment in Japanese young adults. Asian Spine J. 2014;8(5):599.

18. Legaye J, Duval-Beaupère G, Hecquet J, Marty C. Pelvic incidence: a fundamental pelvic parameter for three-dimensional regulation of spinal sagittal curves. Eur Spine J. 1998;7(2):99-103.

19. Wassilew GI, Heller MO, Janz V, Perka C, Müller M, Renner L. High prevalence of acetabular retroversion in asymptomatic adults: a 3D CT-based study. Bone Jt J. 2017;99-B(12):1584-9.

20. Krebs V, Incavo SJ, Shields WH. The anatomy of the acetabulum: what is normal? Clin Orthop. 2009;467(4):868.

21. Diesel CV, Ribeiro TA, Coussirat C, Scheidt RB, Macedo CA, Galia CR. Coxa profunda in the diagnosis of pincer-type femoroacetabular impingement and its prevalence in asymptomatic subjects. Bone Jt J. 2015;97-B(4):478-83.

22. Tian F-D, Zhao D-W, Wang W, Guo L, Tian S-M, Feng A, et al. Prevalence of developmental dysplasia of the hip in Chinese adults: a cross-sectional survey. Chin Med J. 2017;130(11):1261-8.

23. Shon WY, Gupta S, Biswal S, Hur CY, Jajodia N, Hong SJ, et al. Validation of a simple radiographic method to determine variations in pelvic and acetabular cup sagittal plane alignment after total hip arthroplasty. Skelet Radiol. 2008;37(12):1119-27.

24. Eddine TA, Migaud H, Chantelot C, Cotten A, Fontaine C, Duquennoy A. Variations of pelvic anteversion in the lying and standing positions: analysis of 24 control subjects and implications for CT measurement of position of a prosthetic cup. Surg Radiol Anat SRA. 2001;23(2):105-10.

25. Lazennec J-Y, Rousseau M-A, Brusson A, Folinais D, Amel M, Clarke I, et al. Total hip prostheses in standing, sitting and squatting positions: an overview of our 8 years practice using the EOS imaging technology. Open Orthop J. 2015;9:26-44.

26. Grammatopoulos G, Gofton W, Cochran M, Dobransky J, Carli A, Abdelbary H, et al. Pelvic positioning in the supine position leads to more consistent orientation of the acetabular component after total hip arthroplasty. Bone Jt J. 2018;100-B(10): 1280-8.

27. Boese CK, Dargel J, Oppermann J, Eysel P, Scheyerer MJ, Bredow J, et al. The femoral neck-shaft angle on plain radiographs: a systematic review. Skelet Radiol. 2016;45(1):19-28.

28. Fessy MH, Seutin B, Béjui J. Anatomical basis for the choice of the femoral implant in the total hip arthroplasty. Surg Radiol Anat. 1997;19(5):283-6.

29. Beaulé PE, Campbell P, Lu Z, Leunig-Ganz K, Beck M, Leunig M, et al. Vascularity of the arthritic femoral head and hip resurfacing. J Bone Joint Surg Am. 2006;88(Suppl 4):85-96.

30. Scheerlinck T. Cup positioning in total hip arthroplasty. Acta Orthop Belg. 2014;80(3):336-47.

31. Lecerf G, Fessy MH, Philippot R, Massin P, Giraud F, Flecher X, et al. Femoral offset: anatomical concept, definition, assessment, implications for preoperative templating and hip arthroplasty. Orthop Traumatol Surg Res. 2009;95(3):210-9.

32. Pierchon F, Migaud H, Duquennoy A, Fontaine C. Radiologic evaluation of the rotation center of 
the hip. Rev Chir Orthop Reparatrice Appar Mot. 1993;79(4):281-4.

33. Rivière C, Lazennec J-Y, Van Der Straeten C, Auvinet E, Cobb J, Muirhead-Allwood S. The influence of spine-hip relations on total hip replacement: a systematic review. Orthop Traumatol Surg Res. 2017;103(4):559-68.

34. Murphy WS, Yun HH, Hayden B, Kowal JH, Murphy $\mathrm{SB}$. The safe zone range for cup anteversion is narrower than for inclination in THA. Clin Orthop. 2018;476(2):325-35.

35. Upadhyay A, York S, Macaulay W, McGrory B, Robbennolt J, Bal BS. Medical malpractice in hip and knee arthroplasty. J Arthroplast. 2007;22(6 Suppl):2-7.e4.

36. Bsat S, Frei H, Beaulé PE. The acetabular labrum: a review of its function. Bone Jt J. 2016;98-B(6):730-5.

37. Crowninshield RD, Maloney WJ, Wentz DH, Humphrey SM, Blanchard CR. Biomechanics of large femoral heads: what they do and don't do. Clin Orthop. 2004;429:102-7.

38. Matsushita I, Morita Y, Ito Y, Gejo R, Kimura T. Activities of daily living after total hip arthroplasty. Is a $32-\mathrm{mm}$ femoral head superior to a $26-\mathrm{mm}$ head for improving daily activities? Int Orthop. 2011;35(1):25-9.

39. Karimi D, Kallemose T, Troelsen A, Klit J. Hip malformation is a very common finding in young patients scheduled for total hip arthroplasty. Arch Orthop Trauma Surg. 2018;138(4):581-9.

40. Fottner A, Peter CV, Schmidutz F, Wanke-Jellinek L, Schröder C, Mazoochian F, et al. Biomechanical evaluation of different offset versions of a cementless hip prosthesis by 3-dimensional measurement of micromotions. Clin Biomech Bristol Avon. 2011;26(8):830-5.

Open Access This chapter is licensed under the terms of the Creative Commons Attribution 4.0 International License (http://creativecommons.org/licenses/by/4.0/), which permits use, sharing, adaptation, distribution and reproduction in any medium or format, as long as you give appropriate credit to the original author(s) and the source, provide a link to the Creative Commons license and indicate if changes were made.

The images or other third party material in this chapter are included in the chapter's Creative Commons license, unless indicated otherwise in a credit line to the material. If material is not included in the chapter's Creative Commons license and your intended use is not permitted by statutory regulation or exceeds the permitted use, you will need to obtain permission directly from the copyright holder. 